ISSN 2519-7398 (Versión electrónica)

DOI: http://dx.doi.org/10.21704/ac.v78i2.1055

(C) Universidad Nacional Agraria La Molina, Lima - Perú

\title{
Alternativas para estabilizar la Quebrada Cantuta II con fines de mitigación de huaycos en la Universidad Enrique Guzman y Valle
}

\section{Stabilization alternatives at Cantuta II ravine for landslide prevention in Enrique Guzma y Valle University.}

\author{
Carlos Alberto Bravo Aguilar*; Rut Luzmila García Chaca² \\ *Autor de correspondencia
}

\section{Resumen}

El Perú por su ubicación y relieve abrupto, es un candidato idóneo para el desarrollo de huaycos. Los huaycos son eventos geodinámicos cíclicos que, en épocas de lluvias intensas, transportan lodo y escombros por las quebradas, que terminan desembocando en su cono de deyección. Esto representa un peligro constante para las construcciones existentes cercanas al evento. La Universidad Nacional de Educación "Enrique Guzmán y Valle”, está construida dentro del cono aluvial de la quebrada Cantuta II; los sedimentos que llevan los huaycos que descienden por esta quebrada en época lluviosa, se depositan en las instalaciones de la universidad; afectando a la institución con la suspensión de labores académicas y administrativas. De acuerdo a esta problemática se plantea el Estudio de Alternativas para la estabilización geomorfológica de la quebrada Cantuta II. Para tal efecto se inició la investigación con la obtención y extrapolación de data topográfica, geología, estudio de suelos y la hidrológica e hidráulica de flujo de escombros que se emplea para evaluar los tipos de flujos que transportan dichos huaycos. Posteriormente se estudiaron las diversas metodologías existentes, con el propósito de lograr la estabilización de la quebrada, seleccionando las más adecuadas de acuerdo a las características geomorfológicas de zona de estudio. La alternativa propuesta para estabilizar la quebrada Cantuta II consiste en un sistema mixto conformado por: zanjas de drenaje, camas de gravas y diques de piedra, ubicados estratégicamente en la zona alta, media y baja de la quebrada.

Palabras clave: deslizamientos; hidráulica de flujo de escombros; cama de grava; zanja de drenaje; diques de piedra.

Abstract

Peru for its location and abrupt relief, i added to external agents such as the El Niño Phenomenon; is a suitable candidate for the development of landslides, named "huaycos". The huaycos are cyclical geodynamic events that, in times of intense rains, transport mud and debris through the ravines, which end up emptying into their ejection cone. This represents a constant danger for the existing buildings close to the event. The National University of Education "Enrique Guzmán y Valle", is built inside the alluvial cone of the Cantuta II Ravine; The huaycos sediments descend through this ravine in the rainy season, are deposited in the university facilities, which causes flooding in classrooms, tracks, sidewalks, even to the main entrance; Affecting the institution with the suspension of academic and administrative work until the risk of another event happens. According to this problem, the study of alternatives for the geomorphological stabilization of the Cantuta II ravine is proposed. For this purpose, research was started with the collection and extrapolation of topographic and geologic data, study about soil and hydrological and hydraulic study of debris flow used to evaluate the type of flows carried by these huaycos. Subsequently the various existing methodologies were studied, with the purpose of achieving the stabilization of the ravine, selecting the most appropriate ones according to the geomorphological characteristics of the study area. The proposed alternative to stabilize the Cantuta II ravine consists of a mixed system consisting of: drainage ditches, gravel beds and stone dikes, located strategically in the upper, middle and lower part of the ravine.

Keywords: Landslide; debris flow hydraulics; gravel bed; drainage ditch; stone dikes.

\section{Introducción}

Los huaycos son las avenidas torrenciales, que descienden por las quebradas como ríos torrentosos en los que pueden arrastrar pequeñas partículas o bloques rocosos de varias toneladas dependiendo del caudal que tengan (Bravo, 1903). Esto representa un problema para las poblaciones cercanas a la zona del evento; se percibe en los alarmantes datos estadísticos de pérdidas materiales directas e indirectas y de vidas humanas en el Perú, publicadas por el Instituto Nacional de Defensa Civil (INDECI).

En la Universidad Nacional de Educación "Enrique Guzmán y Valle" (UNE), ubicada en el distrito de Chosica; 
los huaycos del 2012 y 2015 dañaron las facultades de Tecnología y Educación Física. En el 2017, se han producido deslizamientos que han interrumpido varias semanas las actividades en dicha casa de estudios. Para entender el mecanismo de estos fenómenos es necesario definir tres zonas de diferentes características en la cuenca de origen, desde el punto topográfico más alto hasta el más bajo: Cuenca de recepción o zona de producción, Canal de escurrimiento o de desagüe, Cono de Deyección. Cuando el transporte de sólidos es grande y la densidad de la mezcla de agua y solidos se eleva por encima de la densidad del agua, las nociones de hidráulica fluvial se desvanecen y se introducen los conceptos de fluidos no - newtonianos. Desde un punto de vista hidráulico, esta rama se conoce como "Hidráulica de Flujo de Escombros".

El objetivo general fue estudiar las alternativas de estabilización de la quebrada Cantuta II con fines de mitigación de huaycos que afectan las instalaciones de la Universidad Nacional de Educación Enrique Guzmán y Valle. Mientras que los objetivos específicos fueron:

- Caracterización de la topografía, geología, hidrología e hidráulica de flujo de escombros en la micro cuenca "Quebrada Cantuta II", para contar con la información que nos permita elegir las alternativas más viables para la estabilización de dicha mico cuenca ante la caída de huaycos..

- Evaluación de las alternativas de estabilización de la micro cuenca "Quebrada Cantuta II" en función de los datos de caracterización; para controlar en forma diferenciada la generación del huayco en las zonas alta, media y baja.

- Analizar y diseñar las alternativas de estabilización escogidas, para dirigir el flujo de agua en forma controlada en la parte superior, controlar la velocidad en la parte media y retener el material arrastrado en la parte baja.

Se realizó un diagnóstico de la micro cuenca "Quebrada Cantuta II" para contar con información topográfica, geológica, hidrológica y de la hidráulica de flujo de escombros; el proceso de dicha información permitirá la evaluación y diseño de las estructuras de control y retención que controlen los huaycos que afectan a la Universidad.

Los estudios de control de huaycos en las quebradas, requieren de una caracterización desde el punto de vista hidrológico, hidráulico de escombros, topográfico, geológico y geotécnico del área en estudio; dicha información nos permitío escoger las alternativas más viables, que varían en cada caso en estudio.

Análisis de información pluviométrica. La determinación del tratamiento de información pluviométrica, obtenida de datos de campo en estaciones meteorológicas, se realiza a través de la metodología del Water Resources Council. Los datos dudosos (outliers) son puntos de información que se alejan significativamente de la tendencia de la información restante. El método de Water Resource Council recomienda una verificación de datos dudosos que consiste en determinar un rango de valores utilizando una ecuación de frecuencia. Los modelos de Gumbel, Pearson, Normal, Log-Normal, Pearson tipo III y Log- Pearson de Tipo III, que son las funciones de distribución de datos, usadas en hidrología para estimar precipitaciones, intensidades o caudales máximos, para diferentes tiempos de retorno mediante la aplicación de modelos probabilísticos. Las pruebas de bondad de ajuste son pruebas de hipótesis que se usan para evaluar si un conjunto de datos es una muestra independiente de la distribución elegida. La prueba de bondad de ajuste más conocida es Kolmogorov - Smirnov. Método por el cual se comprueba la bondad de ajuste de las distribuciones; asimismo, permite elegir la más representativa.

El objetivo del estudio de las precipitaciones, fue la determinación del caudal que circula por el cauce ante determinada intensidad de precipitación; existen métodos diversos para dicho cálculo, pero el que mejor se adapta a cauces pequeños es el Método Racional

$$
Q=C . I . A
$$

Donde el cálculo del caudal es función del coeficiente de escorrentía, la intensidad de precipitación y el área de la cuenca. El Método Racional se usa teniendo en cuenta que producirá resultados aceptables en áreas pequeñas y con alto porcentaje de impermeabilidad, por ello es recomendable que su uso se limite a Cuencas con extensiones inferiores a $200 \mathrm{Ha}$. Dentro de la alternativa para el control de huaycos se encuentran las medidas estructurales, con el objetivo de prevenir o mitigar los desastres ocasionados por el huayco:

a. Zanja de infiltración: Vásquez (2012) señala que las zanjas son construidas de forma transversal a la pendiente misma del terreno, y las características hidráulicas promedio para la sierra peruana son: Pendiente longitudinal cero, ancho de base inferior entre 30 y $50 \mathrm{~cm}$, ancho de bordo superior entre $40-70$ $\mathrm{cm}$, profundidad de zanja entre 30 y $50 \mathrm{~cm}$.

b. Camas de grava: Tienen como función principal aumentar la rugosidad del fondo del cauce en la zona de la quebrada media, bajando la energía cinética del flujo, a fin de que llegue con menor velocidad a los diques de piedra.

c. Diques de Piedra: Los diques serán construidos en la parte media a baja del cauce principal de la quebrada, para su construcción se empleará rocas de la zona que serán colocadas manualmente conformando muros de piedra de gravedad con forma de trapecio.

\section{Localizacion y descripcion del area de estudio.}

Políticamente, la micro cuenca "quebrada Cantuta II", está localizada en la Inter Cuenca Medio Bajo Rímac de la Cuenca del río Rímac, dentro del distrito de LuriganchoChosica, provincia Lima, departamento de Lima. Geográficamente, la micro cuenca en estudio, se encuentra 
localizada entre los puntos extremos identificados por las coordenadas UTM: 8678908,96 N, 315267,86 E, a $1260 \mathrm{msnm}$ y en el extremo inferior $8678980,02 \mathrm{~N}, 314$ $856,78 \mathrm{E}$, a $833 \mathrm{msnm}$. La zona de estudio corresponde a la quebrada Cantuta II, cuyo como cono de deyección se sitúa en las instalaciones de la Universidad Nacional de Educación Enrique Guzmán y Valle. Geológicamente, en la micro cuenca estudiada, se ha encontrado el afloramiento de rocas intrusivas del batolito de la costa, dentro del área predominan dos tipos de unidades lito estratigráficas que se detallara a continuación:

- Súper Unidad Santa Rosa que es la base de la columna geológica de la región Lima, con más de $500 \mathrm{~m}$ de espesor; roca solida volcánica constituyendo a los andes centrales.

- Depósitos Cuaternarios, constituidos por cantos y bloques depositados por el acarreo fluvial, los cuales se constituyen en el material pasible de convertirse en huayco.

\section{Materiales y métodos}

La investigación aplicada busca la generación de conocimiento con aplicación directa a los problemas de la sociedad o el sector productivo. Esta se basa fundamentalmente en los hallazgos tecnológicos de la investigación básica, ocupándose del proceso de enlace entre la teoría y el producto. El presente artículo presenta una visión sobre los pasos a seguir en la solución de un problema en el campus de la Universidad Nacional de Educación Enrique Guzmán y Valle, que periódicamente se ve afectada por huaycos provenientes de la micro cuenca "Quebrada Cantuta II".

Los procesos para lograr los objetivos, comprendieron la búsqueda y recolección de información cartográfica, geológica y pluviométrica, se procesó dicha información con la finalidad de conocer los parámetros técnicos con los que se diseñarón las alternativas de estabilización.

Información Cartográfica fue obtenida de cartas nacionales del Instituto Geográfico Nacional No 19-q, 19-r, 20-q y 20-r en escala 1/5000 del distrito Lurigancho - Chosica donde se encuentra el área de estudio.

El mapa geológico de la zona de estudio fue obtenido de los planos elaborados por el Instituto Geológico Minero y Metalúrgico (INGEMMET) en escala de 1/100 000.

Los datos de precipitaciones fueron obtenidos de la estación meteorológica "Santa Eulalia" del Servicio Nacional de Meteorología e Hidrología del Perú (SENAMHI), y fue seleccionada por las siguientes características: ubicación cerca de la zona de estudio y mayor cantidad de registros anuales completos de precipitación.

El procesamiento de la información cartográfica, para conocer la topografía y relieve de la zona, ha seguido los siguientes pasos:

a. Topografía: La información topográfica de la zona de estudio se obtuvo de los planos del distrito de Lurigancho-Chosica, elaborado por el Instituto

\section{Geográfico Nacional (IGN).}

b. Digitalización de Curvas: Los planos fueron escaneados, para trabajarlos en el programa Photoshop, donde se cambió el color de presentación (a blanco y negro) para realizar las líneas de contorno y comenzar con la digitalización de todas las curvas de nivel de la quebrada Cantuta II y los cauces del torrente de agua.

c. Delimitación de la Quebrada: Las curvas de nivel de la quebrada Cantuta II fueron la herramienta principal para delimitar la micro cuenca que forma ésta quebrada.

d. Con esta información, se dibuja el perfil longitudinal de la quebrada, para identificar zonas con similar pendiente, dividiendo el área de trabajo en zona alta, media y baja, en las que se aplican las distintas alternativas de estabilización en forma diferenciada.

Geología- Geotecnia y Geomorfología: En los mapas geológicos obtenidos, se identifican los estratos geológicos predominantes en la zona de estudio, relacionando dicha información, con la posibilidad de existencia de material suelto, susceptible de ser arrastrado por el caudal de agua.

Geotecnia (Hernandez, 2012): la metodología para la obtención de datos y el procesamento de la información en laboratorio, se detallan a continuación:

- Se registraron seis calicatas estudiadas de $3.00 \mathrm{~m}$ de profundidad, en cada una de los registros se ha clasificado los tipos de suelos encontrados: Grava pobremente gradada con arena y grava arcillosa con arena con clasificación del Sistema Unificado de Suelos GP y GC respectivamente.

- Se seleccionaron muestras alteradas representativas del suelo que se remitieron al laboratorio para los ensayos correspondientes para la su procesamiento

Con esta información, se conocen los parámetros técnicos para el diseño de la cimentación de las estructuras propuestas. Hidrología: La data pluviométrica de la Estación Santa Eulalia fue registrada por el Servicio Nacional de Meteorología e Hidrología (SENAMHI) durante el periodo de 1964-2014. Con esta información se determinaron las precipitaciones máximas en 24 horas mensuales, permitieron estimar los caudales máximos para diferentes tiempos de retorno.

a. Análisis de datos dudosos para las precipitaciones máximas en 24 horas: detección de datos anómalos en series de precipitaciones máximas para periodos de 24 horas, empleando el método de Water Resources Council.

b. Distribución de Probabilidades: Los datos de precipitaciones se emplean para realizar pruebas de distribución: Gumbel, Pearson, Normal, Log-Normal, Pearson tipo III y Log- Pearson de Tipo III.

c. Prueba de Bondad de Ajuste de Precipitaciones Máximas Anuales en 24 horas: La prueba de ajuste de Kolmogorov - Smirnov (distribución Gumbel, Pearson, Normal, Log-Normal, Log- Pearson de Tipo III). 
d. Curva de Intensidad - Duración - Frecuencia (IDF): El dato de lluvias máximas se emplea para graficar las curvas de Intensidad-Duración-Frecuencia y calcular la ecuación de intensidad en función del tiempo de retorno y la duración de la tormenta:

$$
I=\frac{10^{k} T^{m}}{t^{n}}
$$

Dónde:

$\mathrm{I}=$ Intensidad máxima, en $(\mathrm{mm} / \mathrm{h})$

$\mathrm{T}=$ Periodo de retorno (años)

$\mathrm{t}=$ Duración de precipitación (min)

e. Tiempo de concentración: El tiempo de concentración se obtiene mediante la fórmula propuesta por Kirpich, la cual relaciona la longitud máxima de salida con la pendiente de la superficie para obtener el tiempo de concentración.

$$
x=\frac{0,06628 \cdot L^{0,7}}{S^{0,385}}
$$

f. Caudal de diseño: Para la estimación del caudal se utiliza el método racional ya que se tiene referencias para áreas pequeñas es recomendable su uso.

$$
Q=\frac{C I A}{3,6}
$$

$\mathrm{Q}=$ Caudal $\left(\mathrm{m}^{3} / \mathrm{s}\right)$

$\mathrm{I}=$ Intensidad máxima $(\mathrm{mm} / \mathrm{h})$

$\mathrm{A}=$ Área de drenaje $\left(\mathrm{km}^{2}\right)$

$\mathrm{C}=$ Coeficiente de escorrentía.

Hidráulica de Flujo de Escombros: Estudio de las avenidas con torrentes; permite determinar parámetros característicos del flujo, tales como: caudal total, volumen de escombros, densidad del flujo y velocidad del flujo:

a. Concentración de Sedimentos (Cv): Indica el comportamiento del flujo, fue calculado siguiendo los lineamientos de la clasificación propuesta por O'Brien y Julien (1988).

b. Volumen de Agua: se estima mediante el producto del caudal de agua seleccionado para un tiempo de retorno escogido de acuerdo al tiempo de concentración.

$$
V_{a}=Q^{*} T c
$$

Donde:

Q: Caudal de agua $\left(\mathrm{m}^{3} / \mathrm{s}\right)$ :

$\mathrm{T}_{\mathrm{c}}$ : Tiempo de concentración ( $\mathrm{s}$ ) c. Volumen de Sedimentos: El volumen de sedimentos se estima mediante el valor de la concentración de sedimentos:

$$
C_{v}=\frac{V_{s}}{V_{s}+V_{a}} \quad \frac{C_{v} V_{a}}{1-C_{v}}=V_{s}
$$

Donde:

$\mathrm{V}_{\mathrm{s}}$ : Volumen de sedimentos $\left(\mathrm{m}^{3}\right)$

$\mathrm{V}_{\mathrm{a}}$ : Volumen de agua $\left(\mathrm{m}^{3}\right)$

d. Cálculo del caudal total de flujo: El caudal total de flujo se calcula empleando el método de JICA, basado en los desarrollos de Takahashi $(1978,1981)$ para determinar el caudal máximo de la corriente de detritos.

$$
Q_{d}=Q_{d} P=Q_{0} \frac{C^{*}}{C^{*}-C_{d}}
$$

Donde:

$\mathrm{Q}_{\mathrm{d}}$ : Caudal máximo del flujo de detritos

$\mathrm{Q}_{\mathrm{o}}$ : Caudal máximo líquido

$\mathrm{C}_{\mathrm{d}}$ : Concentración volumétrica de sólidos

$\mathrm{C}^{*}$ : Concentración máxima de los sólidos.

e. Densidad del flujo: Para su estimación, se usa una variación de la fórmula para cálculo de caudales propuesta por Takahashi.

$$
Q_{t}=\left[\left(1+\frac{V s}{V a}\right)+\frac{(\rho-1)}{(\sigma-\rho)}\right] * Q_{l}
$$

Donde:

$Q_{t}$ : Caudal total o caudal máximo de flujo de detritos $\mathrm{V}_{\mathrm{s}}$ : Volumen de detritos $\left(\mathrm{m}^{3}\right)$

Va: Volumen del agua $\left(\mathrm{m}^{3}\right)$

$\rho:$ Densidad del fluido de detritos $\left(\mathrm{t} / \mathrm{m}^{3}\right)$

$\sigma:$ Densidad del sedimento $\left(\mathrm{t} / \mathrm{m}^{3}\right)$

$\mathrm{Q}_{1}$ : Caudal líquido $\left(\mathrm{m}^{3} / \mathrm{s}\right)$

f. Velocidad del flujo: La velocidad de flujo es estimada empleando la fórmula propuesta por Costa (1984):

Donde:

$$
V=2.11 * Q^{0.33} * S^{0.33}
$$

$\mathrm{V}$ : Velocidad $(\mathrm{m} / \mathrm{s})$

Q: Caudal de flujo de detritos $\left(\mathrm{m}^{3} / \mathrm{s}\right)$

S: Pendiente.

Diseño de la Alternativa Elegida: Para el diseño de la alternativa mixta de estabilización, se tomaron en cuenta las siguientes alternativas: 
- Zanjas de infiltración en la parte alta de la micro cuenca, para dirigir el flujo de agua, con baja velocidad hasta el cauce principal. En los diseños de dichos canales se corroboran las dimensiones del canal y velocidad de arrastre de partículas.

El caudal de diseño de cada zanja se calcula a partir del área que estaba por encima de la zanja por la lámina máxima de precipitación de 24 horas para el periodo de retorno de 200 años:

$$
Q_{z}\left(\frac{m^{3}}{s}\right)=\operatorname{Lamina}\left(\frac{l}{h}\right) x \operatorname{area} \frac{\left(\mathrm{m}^{2}\right)}{24 * 1000 * 3600}
$$

Para el trazo de una zanja se tiene como referencia las direcciones de la curva de nivel la pendiente variaba de 3 a $20 \%$ que va dependiendo de la longitud de la zanja.

Una vez obtenidos los caudales de cada zanja se procede al diseño hidráulico que nos proporciona datos de velocidades, tirante, radio hidráulico, etc.

Camas de grava en la parte media del cauce, para aumentar la rugosidad de fondo y disminuir la energía cinética del flujo; este procedimiento es empírico, no hay un procedimiento matemático que permita su verificación, sin embargo hay experiencias exitosas de su uso en el control de huaycos.

Diques de piedra en la parte media - baja del cauce, para detener el flujo de solidos que ya vienen con baja velocidad y permitir solo el paso del agua.

Para su dimensionamiento apropiado es necesario conocer ciertos parámetros básicos como:

- Densidad específica: 1.6 ton $/ \mathrm{m}^{3}$ calculada con el flujo de escombros que servirá al cálculo de los empujes superficiales.

- El ángulo de fricción: $32^{\circ}$ que se obtuvo del estudio geotécnico.

- Cohesión $0.16 \mathrm{~kg} / \mathrm{cm}^{2}$ y la capacidad de carga admisible es de $4.07 \mathrm{~kg} / \mathrm{cm}^{2}$ ambos datos fueron obtenidos del estudio geotécnico.

\section{Resultados y discusión}

Cumpliendo con el primer objetivo específico del presente trabajo, se procede a la caracterización de la micro cuenca en estudio; es el primer paso para escoger las alternativas técnicas de estabilización ante la presencia de huaycos.

De la caracterización, topográfica, se han determinado las características fisiográficas indicadas en la Tabla 1; en el área, se ha estudiado las pendientes del cauce principal de la micro cuenca, con el fin de identificar zonas de pendiente similar en las que se pueda aplicar las alternativas de estabilización diferenciadas.
Tabla 1. Parámetros Fisiográficos de la Quebrada

\begin{tabular}{lcccccc}
\hline \multirow{2}{*}{$\begin{array}{l}\text { Micro } \\
\text { cuenca }\end{array}$} & \multicolumn{5}{c}{ Características fisiográficas de la microcuenca } \\
\cline { 2 - 7 } & $\begin{array}{c}\text { Área Perímetro Longitud } \\
\mathrm{km}^{2}\end{array}$ & $\mathrm{~km}$ & $\begin{array}{c}\text { Cota } \\
\text { mm }\end{array}$ & $\begin{array}{c}\text { Cota } \\
\text { m.s.n.ma máxima }\end{array}$ & $\begin{array}{c}\text { Pendiente } \\
\text { m.s.n.m }\end{array}$ & $\begin{array}{c}\text { Promedio } \\
(\mathrm{m} / \mathrm{m})\end{array}$ \\
\hline $\begin{array}{l}\text { Quebrada } \\
\text { Cantuta 2 }\end{array}$ & 0,402 & 2,44 & 1,72 & 840,00 & 1280 & 0,307 \\
\hline
\end{tabular}

A continuación se muestra los datos de cálculo de las pendientes del cauce principal; identificando tres zonas con pendientes similares dentro del área de estudio.

Tabla 2. Características de las sub cuenca Cantuta II

\begin{tabular}{lll}
\hline Sub cuenca & Área $\left(\mathrm{km}^{2}\right)$ & Pendiente $\%$ \\
\hline Alta & 0,126 & 50,7 \\
Media & 0,202 & 42,3 \\
Baja & 0,074 & 27,47 \\
\hline
\end{tabular}

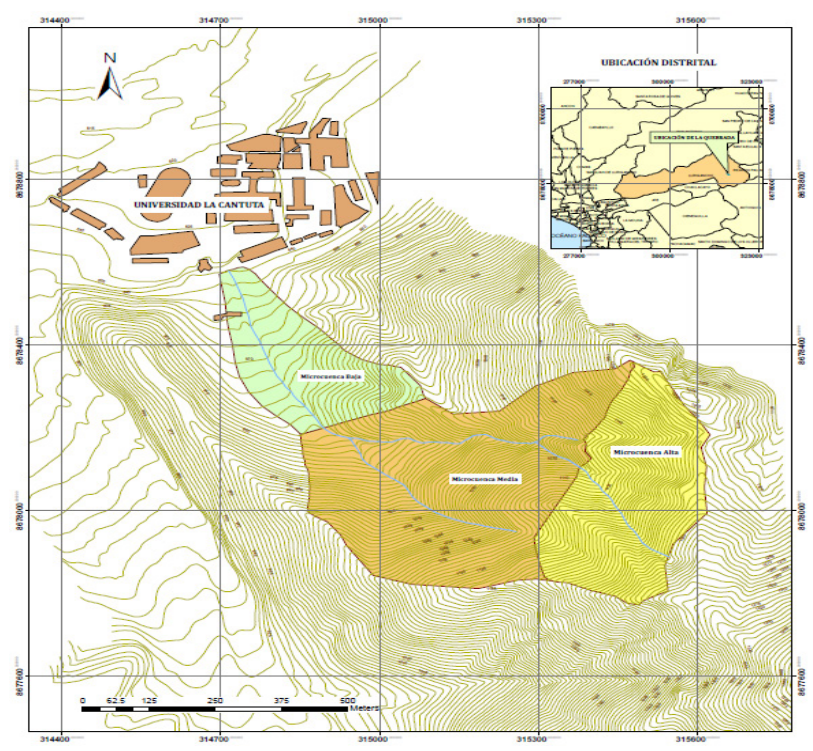

Figura 1. Plano de planta de la sub cuenca Cantuta II.

Geológicamente, dentro del área predominan dos tipos de unidades lito estratigráficas que se detallara a continuación:

- Súper Unidad Santa Rosa

- Depósitos Cuaternarios

Del estudio de mecánica de suelos y geotécnica, realizado como parte del diagnóstico de la micro cuenca; en la Tabla 3, se presentan los resultados de los ensayos estándar; el objetivo es determinar la clasificación de los suelos presentes en la superficie, con el fin de definir la clasificación del material; esta clasificación nos permite confirmar la composición de suelos que es pasible de ser sometida al arrastre del agua, conformando los huaycos. Un suelo con clasificación GP o GC, como los encontrados, es granular, con muy poca cohesión es decir, no existe un efecto cementante entre las partículas que permita atenuar el efecto de arrastre del agua en las partículas. 
Tabla 3. Resumen de los ensayos estándar de Clasificación de suelos

\begin{tabular}{ccccccccc}
\hline & & & \multicolumn{3}{c}{ Granulometría } & \multicolumn{2}{c}{ Límites de } & \multirow{2}{*}{ Código } \\
\cline { 4 - 7 } & Prof. (m) & \multirow{2}{*}{ SUCS } & Grava & Arena & Fino & & Atterberg & Descripción \\
\cline { 4 - 7 } & & & $\%$ & $\%$ & $\%$ & LL & LP & \\
\hline C-1 & $0,80-3,00$ & GP & 73,50 & 24,20 & 2,30 & - & NP & 1,12 Grava pobremente gradada con arena \\
E-2 & $1,50-3,00$ & GC & 59,70 & 25,50 & 14,80 & 27,00 & 19,00 & 7,59 Grava arcillosa con arena \\
E-4 & $1,00-3,00$ & GC & 56,90 & 23,80 & 19,30 & 19,00 & 15,00 & 10,95 Grava arcillosa con arena
\end{tabular}

Fuente: Estudio de Pre inversión a Nivel de Perfil de la Instalación del Servicio de Protección Contra Inundaciones del Cauce de la Quebrada de Santo Domingo de la UNE.

Con la finalidad de determinar los parámetros de resistencia ( с у Ø), se ha previsto la ejecución de 01 ensayo de corte directo, en suelos, cuyos resultados están en la Tabla 4.

Tabla 4. Resumen Ensayo de Corte Directo.

\begin{tabular}{ccccc}
\hline Código & Muestra & $\begin{array}{c}\text { Clasificación } \\
\text { SUCS }\end{array}$ & $\begin{array}{c}\text { Ángulo de } \\
\text { fricción } \mathrm{f}\end{array}$ & $\begin{array}{c}\text { Cohesión C } \\
\left(\mathrm{Kg} / \mathrm{cm}^{2}\right)\end{array}$ \\
\hline E-2 & $\mathrm{M}-1$ & $\mathrm{GC}$ & $31^{\circ}$ & 0,16 \\
\hline
\end{tabular}

Fuente: Estudio de Pre Inversión a Nivel de Perfil de la Instalación del Servicio de Protección Contra Inundaciones del Cauce de la Quebrada de Santo Domingo de la UNE

La capacidad de carga admisible del suelo es $\mathrm{Q}_{\mathrm{adm}}=4,07$ $\mathrm{Kg} / \mathrm{cm}^{2}$ considerando un factor de seguridad $\mathrm{Fs}=3$.

De los resultados anteriores, confirmamos que existe material suelto granular en la micro cuenca estudiada, que será arrastrado por las aguas conformando los huaycos que afectarán al campus de la UNE; también se ha confirmado la capacidad portante de los suelos, que servirá para el dimensionamiento de las estructuras de retención de material del huayco.

Los rasgos geomorfológicos presentes en la zona de estudio son los siguientes:

- Cauce Actual: El cauce principal es la ruta por donde circula la escorrentía, formando un relieve con pendiente aproximada de $45 \%$, en forma casi simétrica de bordes escarpados y donde sobresalen acumulaciones de bloques que aparentemente forman un relieve semi abrupto. El cauce principal de la quebrada mide 1,73 $\mathrm{km}$.

- Conos de Deyección: Se ubican en la parte inferior de la quebrada Cantuta II, sobre un relieve de baja pendiente, con valores entre los $10^{\circ}$ y $20^{\circ}$, en forma de un abanico y con cauces no definidos, actualmente en el cono de deyección de la quebrada se asienta la UNE.

El objetivo de la información geomorfológica es la de identificar las zonas donde se aplicaran los distintos tratamientos para el control de huaycos en la zona; en el cauce principal el objetivo es disminuir la energía cinética del flujo de escombros, mientras que en el cono de deyección, la meta es retener dichos flujos, que ya vienen con velocidad controlada, para evitar que dicho material, dañe las instalaciones de la UNE.

El objetivo del estudio hidrológico, como parte de la caracterización de la micro cuenca, es la de conocer el caudal de agua para distintos tiempos de retorno y diferentes intensidades, Análisis de los datos Dudosos para Precipitaciones Máximas Anuales en 24 horas: La data analizada consta de 51 valores de precipitaciones máxima en 24 horas, que arrojaron como resultados a 10,59 de promedio aritmético y 9,22 desviación estándar.

a. Prueba de Bondad de ajuste de precipitaciones máximas Anuales en 24 horas.

De la prueba de bondad de ajuste de Kolmorogv Smirnov se obtiene las órdenes de ajuste para todas las distribuciones empleadas, siendo el $\mathrm{N}^{\circ} 1$ Log- Pearson de tipo III el mejor ajuste de todas.

b. Coeficientes de Distribución Regional: Se halló a 5a como la región hidrológica de la zona de estudio. Finalmente, luego del cálculo de los parámetros se consiguió los coeficientes regionales para determinar precipitaciones e intensidades para diferentes tiempos de retorno.

c. Tiempo de Concentración: El tiempo de concentración de la tormenta estimado fue de 0.187 horas, según la ecuación de Kirpich.

$$
t c=\frac{0,06628 \cdot L^{0,77}}{S^{0,385}}
$$

d. Caudal de Diseño: El caudal de escorrentía calculado por el método racional fue estimado para diferentes tiempos de retorno e intensidades halladas en el ítem anterior. Se ha escogido un periodo de retorno de 200 años, con una intensidad de precipitación de $2,29 \mathrm{~mm} /$ hr, Coeficiente de escorrentía de 0,8 y el caudal de 0,20 $\mathrm{m}^{3} / \mathrm{seg}$.

El caudal de agua obtenido, ingresa como dato en el cálculo del flujo de escombros. El objetivo es la caracterización del huayco, conociendo el caudal total de agua y sólidos, velocidad, y densidad.

La culminación del proceso de caracterización de los huaicos, se determina con el estudio del flujo de escombros; la combinación del agua con los materiales solidos presentes en la superficie estudiada, nos da los parámetros que se utilizan en el diseño de las estructuras de control de material arrastrado.

a) Concentración de Sedimentos $(\mathrm{Cv})$

La concentración de sedimentos es 0,450, fue seleccionada de la Tabla clasificación de O'Brien y Julien (1988).

b) Volumen de Agua 
El volumen de agua obtenido es:

$$
V_{a}=Q^{*} T c---->V_{a}=0,2 * 0,18 * 3600=129,6 \mathrm{~m}^{3} \text { por }
$$

Donde:

$\mathrm{Q}\left(\mathrm{m}^{3} / \mathrm{s}\right)$ : Caudal de agua

$\mathrm{T}_{\mathrm{c}}(\mathrm{s})$ : Tiempo de concentración

c) Volumen de sedimentos

De la concentración de sedimentos y el volumen de agua estimado encontramos el volumen de sedimentos a partir de:

$$
\frac{C_{v} V_{a}}{1-C_{v}}=V_{s} \longrightarrow \frac{0,45 * 129,6}{1-0,45}=106,03 \mathrm{~m}^{3}
$$

\section{Caudal total del flujo}

El caudal de total de flujo de escombros estimado fue de $0,61 \mathrm{~m}^{3} / \mathrm{s}$, obtenido por la siguiente expresión, Takahashi $(1978,1981)$.

$$
Q_{d}=Q_{0} \frac{C^{*}}{C^{*}-C_{d}}=0.2 \frac{0,65}{0,65-0,45}=0,61 \mathrm{~m}^{3} / \mathrm{s}
$$

Donde $Q_{d}$ representa el caudal máximo del flujo de detritos, $\mathrm{Q}_{\mathrm{o}}$ el caudal máximo líquido, $\mathrm{C}_{\mathrm{d}}$ concentración volumétrica de sólidos y $\mathrm{C}^{*}$ la concentración máxima de los sólidos generalmente toma el valor de $65 \%$.

\section{Densidad de Flujo}

La obtenida del flujo fue de $1.6 \mathrm{t} / \mathrm{m}^{3}$ que fue obtenida de la variación de la fórmula para cálculo de caudales propuesta por Takahashi, que tendría como única incógnita a la densidad del flujo tal como se muestra a continuación:

$$
Q_{t}=\left\lfloor\left(1+\frac{V s}{V a}\right)+\frac{(\rho-1)}{(\sigma-\rho)}\right\rfloor * Q_{l}
$$

Donde:

$\mathrm{Q}_{\mathrm{t}} \quad=0,61 \mathrm{~m}^{3} / \mathrm{s}$ es el caudal total o caudal máximo de flujo de detritos

$\mathrm{V}_{\mathrm{s}}\left(\mathrm{m}^{3}\right)=106,03$ es el volumen de detritos,

$\mathrm{Va}\left(\mathrm{m}^{3}\right)=129,6$ volumen del agua

$\rho\left(t / \mathrm{m}^{3}\right)=$ densidad del fluido de detritos, es la incógnita por despejar.

$\sigma\left(\mathrm{t} / \mathrm{m}^{3}\right)=1,8$ por lo general toma este valor la densidad del sedimento

$\mathrm{Q}_{1}\left(\mathrm{~m}^{3} / \mathrm{s}\right)=0,2$ es el caudal líquido.

$$
0.61=\left[\left(1+\frac{106.03}{129.6}\right)+\frac{(\rho-1)}{(1.8-\rho)}\right] * 0.2 \rightarrow \rho=1,59 \mathrm{~T} / \mathrm{m}^{3}
$$

\section{Velocidad de Flujo}

La velocidad del flujo de escombros estimada fue de 0,94 $\mathrm{m} / \mathrm{s}$ y se encuentra dentro del rango del flujo de escombros que va de 0,5 a $20 \mathrm{~m} / \mathrm{s}$ propuesto por el autor Costa (1984) tal como se en la siguiente expresión:

$V=2,11 * Q^{0,33} * S^{0,33} \rightarrow V=2,11 \times 0,6^{0,33} \times 0,30,33=1,21 \mathrm{~m} / \mathrm{s}$

Donde:

Q $\left(\mathrm{m}^{3} / \mathrm{s}\right)$ : Caudal de flujo de detritos

S : Pendiente

Todos los cálculos previos, corresponden a la caracterización de la micro cuenca "Quebrada Cantuta II"; se ha definido las zonas de similar pendiente en la micro cuenca; de la información geológica y geotécnica, confirmamos la presencia de material superficial pasible de arrastre por las aguas; con los cálculos hidrológicos, determinamos el caudal de agua presente debido a las precipitaciones pluviales y con la hidráulica del flujo de escombros, conocemos el caudal, la velocidad, la densidad y el volumen del huayco.

Se ha identificado tres zonas, las cuales tienen un tratamiento específico cada una; no se puede pretender usar una sola solución para toda el área, dado que los efectos geodinámicos son distintos en la zona alta, media y baja.

En la zona alta, ante el evento de una fuerte precipitación, se inicia el movimiento de las partículas de suelo presentes en la superficie, las cuales son arrastradas hacia el cauce central.

En la zona media, debido a la pendiente, dichas partículas incrementan su energía cinética, aumentando su velocidad al discurrir por el cauce principal de la micro cuenca, arrastrando más material que se encuentre en dicho canal.

En la zona baja, el material arrastrado, llega con máxima velocidad, abriéndose paso por el cono de deyección y dañando las instalaciones de la Universidad que allí se encuentran.

Se pretende, en la zona alta, dirigir el flujo de precipitaciones hacia el cauce principal en forma controlada, dirigiendo el flujo mediante zanjas de poca pendiente, impidiendo que se inicie el movimiento de escombros; en la zona media, el objetivo es bajar la velocidad de flujo, disminuyendo su energía cinética, y en la parte baja, se trata de detener el flujo de escombros, permitiendo solo el paso del agua.

Las zanjas de infiltración o mejor llamadas de recolección se colocarán en la parte alta a media de la micro cuenca y serán trazadas hasta el cauce principal de la quebrada. Tendrán medidas preliminares de 30 x 40, 20 x $30 \mathrm{~cm}$ de profundidad y ancho respectivamente,

En la Tabla 5 se muestra el resumen de los datos calculados como caudal, pendiente, longitud, tirante, velocidad para 6 de las 12 zanjas diseñas. 
Se colocará enrocado en forma de camas en tramos de la parte media del cauce principal de la quebrada, se fijaran manualmente en filas una encima de otra, sin ningún tipo de mortero los diámetros mínimos y máximos de las rocas son de 10 a $30 \mathrm{~cm}$.

La longitud máxima es de 6 metros y el ancho será colocado de acuerdo al lecho de la quebrada, con una altura no mayor a 0,40 metros.

La función de dichos elementos es la de aumentar la fricción, disminuyendo de esta manera la velocidad del flujo de escombros.

Se trata de una medida empírica, no hay antecedentes de cálculo de dichos elementos, sin embargo, ha sido utilizada con éxito, como medidas complementarias de estabilización de micro cuencas y sub cuencas.

Diques de piedra: Se propone nueve diques de piedra que serán construidos en la parte media a baja del cauce principal de la quebrada, para su construcción se empleara rocas de la zona que serán apiladas, conformando bloques trapezoidales, a la manera de un muro de gravedad.

Los diques fueron diseñados para 2 y 3 metros de altura suponiendo inicialmente medidas provisionales geométricas a ser verificadas con el cálculo de estabilidad al volteo y deslizamiento.

Los resultados de diseño de los diques de piedra, se muestran a continuación en la Tabla 6 .

Tabla 5. Datos Calculados de las zanjas de Infiltración

\begin{tabular}{lllllllllll}
\hline \multicolumn{2}{c}{$\begin{array}{c}\mathrm{N}^{\circ} \text { de } \\
\text { Tramo Inicial }\end{array}$} & $\begin{array}{c}\text { Cota } \\
\text { Final }\end{array}$ & \multicolumn{2}{c}{ Longitud Pendiente } & Área & $\begin{array}{c}\mathrm{Q} \\
(\mathrm{m} 3 / \mathrm{s})\end{array}$ & $\begin{array}{c}\text { Tirante } \\
(\mathrm{m})\end{array}$ & $\begin{array}{c}\mathrm{V} \\
(\mathrm{m} / \mathrm{s})\end{array}$ \\
\hline 1 & 1231 & 1225 & 219,61 & 0,03 & 7263,8 & 0,005 & 0,12 & 0,103 \\
2 & 1224 & 1195 & 313,90 & 0,09 & 11556,8 & 0,007 & 0,10 & 0,16 \\
3 & 1230 & 1167 & 390,58 & 0,16 & 16312,0 & 0,010 & 0,11 & 0,22 \\
4 & 1215 & 1140 & 401,65 & 0,14 & 16320,0 & 0,010 & 0,10 & 0,24 \\
5 & 1185 & 1115 & 339,10 & 0,20 & 13783,9 & 0,009 & 0,09 & 0,24 \\
6 & 1150 & 1093 & 267,80 & 0,20 & 12811,4 & 0,008 & 0,08 & 0,23 \\
\hline
\end{tabular}

Tabla 6. Datos Calculados de diques de piedra

\begin{tabular}{lcccccc}
\hline $\begin{array}{c}\text { Muro } \\
\text { tipo }\end{array}$ & $\begin{array}{c}\text { Altura } \\
(\mathrm{m})\end{array}$ & $\begin{array}{c}\text { Base } \\
\text { mayor(m) }\end{array}$ & $\begin{array}{c}\text { Base } \\
\text { menor } \\
(\mathrm{m})\end{array}$ & $\begin{array}{c}\text { Empuje } \\
\text { activo } \\
(\mathrm{Kg})\end{array}$ & FSD & FSV \\
\hline M1 & 2,00 & 1,50 & 0,30 & 831,67 & 2,631 & 7,139 \\
M2 & 3,00 & 2,90 & 0,40 & 1871,51 & 3,491 & 12,091 \\
\hline
\end{tabular}

\section{Conclusiones}

Se ha logrado caracterizar la micro cuenca "Quebrada Cantuta II", habiéndose determinado 3 zonas de estudio: Alta media y baja, con pendientes similares; se conoce el material predominante en la zona, el que es susceptible de ser arrastrado por el agua; se ha determinado el caudal de flujo de escombros

Con los datos hidrológicos y de cálculo del caudal de agua, se ha definido el caudal de flujo de escombros en $0.6 \mathrm{~m} 3 / \mathrm{s}$ con densidad de 1.59 ton $/ \mathrm{m} 3$, para la quebrada Cantuta II.
Se han propuesto las alternativas de solución en forma diferenciada para las tres zonas identificadas en la micro cuenca, disminuyendo la velocidad y erosión hídrica en la parte alta, controlando la velocidad del flujo de escombros en la parte media y reteniendo el paso de los escombros en la parte baja.

En la zona alta, se propone 12 zanjas de recolección o drenaje derivando el agua al cauce principal, en la parte media, se propone enrocado colocado manualmente en una longitud de $510 \mathrm{~m}$, para aumentar la rugosidad del cauce y en la parte baja se deberían construir nueve diques de mampostería de piedra, para retención de los escombros, y no alcancen las instalaciones de la Universidad Nacional de Educación (UNE).

\section{Literatura citada}

Bravo, J. 1903. Los Huaycos. Vol. Informe Memorias Sociedad de Ingenieros del Perú. V:13-21. Lima, Perú.

Hernandez, J. 2012. Riesgos Geológicos Externos. Tomado de: http://es.slideshare.net/Alberkar/riesgos-geolgicosexternos-2012.

O'Brien, J.S. \& Pierre y Julien. 1988. Laboratory Analysis of Mudflow Properties. Joumal of Hydraulic Engineering, 114 (8), ASCE.

Vásquez. 2012. Cosecha de Agua de lluvia en laderas Semiáridas de la sierra y su impacto en el proceso de Desertificación y cambio Climático. Libro UNALM Lima, Perú Pag. 25-26. 